\section{The dangers of DNA vaccination}

To the editor-DNA vaccination has entered clinical trials, and may ultimately be used on a large scale. It involves the injection of naked DNA that encodes antigens under the control of a eukaryotic promoter, and results in strong and sustained humoral and cell-mediated responses. The generic nature of the technology will facilitate the development of new vaccines; DNA vaccines are stable and will not require continuous cold storage. These properties are likely to lead to extremely widespread clinical and agricultural use in both the developed and developing world. In the developing world, inadequate health care resources will make post-vaccination control difficult. In this context, safety must be a particular concern.

After injection, some DNA may persist and reach distant sites. Although the amount of DNA uptake by distant cells is not large, it is unlikely to be zero. Moreover, DNA injected intravenously into pregnant mice reaches fetuses ${ }^{1}$. If after vaccination DNA is taken up by fetal or germ-line cells, immunological tolerance may be induced in the progeny (and descendants) of the vaccinated individual. In hepatitis B patients infected at birth, neonatal tolerance plays an important part in virus persistence ${ }^{2}$. Transgenic mice are often tolerant to transgene-encoded proteins; for example, expression of Schistosoma mansoni glutathione Stransferase leads to tolerance and to more severe experimental infections ${ }^{3}$. However, in mice that express the transgene extrathymically and relatively late, tolerance may not be induced. Current approaches to DNA vaccination use the strongest available promoters, usually the cytomegalovirus enhancer-promoter, to drive expression of antigen genes. In transgenic mice, this promoter is active during development $^{4}$ and in the thymus ${ }^{5}$.

Tolerance resulting from DNA vaccination would produce consequences both for the individuals and for the population, as tolerant individuals are expected to be more susceptible to infection and/or they may become carriers, a potentially much more serious problem. Possible solutions include the use of promoters that are both musclespecific and completely inactive during prenatal and early post-natal life, and systems that rely on the expression of a ligand-regulatable transactivator, in which case tolerance induction would require the expression of transactivator and presence of the ligand as well as the plasmid that encodes the antigen. Whatever the magnitude of

this potential problem, it should be taken into account in the design and testing of widespread.

\section{D.C. Górecki \& J. PAUl Simons}

Department of Anatomy and Developmental Biology, Royal Free and University College Medical School, Royal Free Campus Rowland Hill Street, London NW3 2PF UK E-mail:simons@rfhsm.ac.uk vaccines before DNA vaccination becomes

\title{
Diabetes and the risk of miscarriage
}

To the editor-In an unworthy parroting of an assertion by Moley and colleagues ${ }^{1}$, placed prominently on the first contents page [and highlighted on the cover, no less...Ed.] of the December issue, we read that "diabetes in early pregnancy is associated with a high risk of miscarriages...."

Twelve years ago I tried to put this erroneous notion to rest ${ }^{2}$. Your report was picked up by Lancet $^{3}$, where it might lead to diabetic women being needlessly terrified. Furthermore, the inference by Moley et al., thoughtlessly repeated on your contents page, that their findings regarding blastocyst-stage embryos might be relevant to fetal maldevelopment, is also nonsensical ${ }^{4}$.

It is amazing that in our age of instantaneous and exhaustive retrieval of medical literature there should be the bibliographic laxity frequently encountered, seemingly greater than in the past, for reasons better left to editors to ponder.

\section{H. KALTER}

Children's Hospital Research Foundation

3333 Burnet Avenue

Cincinnati, OH 45229, USA

email Kalter@email.uc.edu

Moley et al. reply-We thank Dr. Kalter for his interest in our paper. In his 1987 review, he concluded "it is most probable that there is no excess of spontaneous abortions in the diabetic pregnancy." Fortunately, in the intervening twelve years, several welldesigned clinical studies investigating diabetes in pregnancy have refuted Kalter's conclusion. The most definitive evidence is the Diabetes in Early Pregnancy study ${ }^{5}$ conducted by the National Institute of Child Health and Human Development. In this prospective, multicenter, collaborative study - the largest of its kind so far-the authors definitively establish a direct correlation between spontaneous abortion in
1. Tsukamoto, M. et al. Gene transfer and expression in progeny after intravenous DNA injection into pregnant mice. Nature Genet. 9, 243-248 (1995).

2. Chisari, F.V. \& Ferrari C. Hepatitis B virus immunopathogenesis. Annu. Rev. Immunol. 13, 29-60 (1995).

3. Xu, X. et al. Expression of a Schistosoma mansoni 28-kilodalton glutathione S-transferase in the livers of transgenic mice and its effect on parasite infection. Infect. Immunol. 65, 3867-3874 (1997).

4. Baskar, J.F. et al. Developmental analysis of the cytomegalovirus enhancer in transgenic animals. I Virol. 70, 3215-3226 (1996)

5. Baskar J.F. et al. The enhancer domain of the human cytomegalovirus major immediate-early promoter determines cell type-specific expression in transgenic mice. J. Virol. 70, 3207-3214 (1996).

women with insulin-dependent diabetes mellitus and glycosylated hemoglobin levels, a marker for glucose control. Women with diabetes in poor metabolic control show an increase risk of pregnancy loss. Several other clinical studies, as well as animal studies, concur with these findings $s^{6,7}$.

We note that Kalter does not question our experimental results. In our article, we provided clear evidence to support a deleterious effect of maternal diabetes and elevated glucose levels per se on the preimplantation murine blastocyst. Our findings describe a biochemical effect and suggest a precise molecular mechanism to explain a causeand-effect relationship between maternal diabetes and spontaneous abortion and fetal malformations. Contrary to Kalter's assertion, similar phenomena, arrived at by different mechanisms in the blastocyst model, have been shown to result in miscarriage and fetal malformations ${ }^{8}$.

Finally, even if this issue were equivocal, and we believe that it is not, it would still be prudent to err on the side of caution in counseling diabetic patients. Therefore, any women reading about our report, rather than being terrified, should be at ease in the knowledge that by practicing careful metabolic control, they can minimize the risk of pregnancy loss.

Kelle Moley ${ }^{1} \&$ Mike Mueckler ${ }^{2}$ ${ }^{1}$ Department of Obstetrics and Gynecology, Washington University School of Medicine 499 S. Euclid Ave., St. Louis, MO 63110 ${ }^{2}$ Department of Cell Biology and Physiology, Diabetes Research and Training Center, Washington University School of Medicine 660 S. Euclid Ave., St. Louis, MO 63110 emailmike@cellbio.wustl.edu

1. Moley, K.H., Chi, M.M.-Y., Knudson, C.M., Korsmeyer, S.J. \& Mueckler, M.M. Hyperglycemia induces apoptosis in the pre-implantation embryos through cell death effector pathways. Nature Med. 
4, 1421-1424 (1998).

2. Kalter, H. Diabetes and spontaneous abortion: a historical review. Am. J. Obst. Gyn. 156, 1243-1253 (1987)

3. McCarthy, M. Hyperglycaemia triggers embryonic programmed cell death. Lancet 352, 1911 (1998).

4. Kalter, H. Reproductive toxicology in animals with induced and spontaneous diabetes. Reprod. Toxicol. 10, 417-438 (1996).
5. Mills, J.L. et al. Incidence of spontaneous abortion among normal women and insulin-dependent diabetic women whose pregnancies were identified within 21 days of conception. N. Engl. J. Med. 319, 1617-1623 (1988).

6. Sutherland, H.W. \& Pritchard, C.W. Increased incidence of spontaneous abortion in pregnancies complicated by maternal diabetes mellitus. Am. J. Obstet. Gynecol. 156, 135-138 (1987).
7. Moley, K.H., Diamond, M.P., Vaughn, W.K. \& DeCherney, A.K. Effect of diabetes mellitus on mouse pre-implantation embryo development. /. Reprod. Fertil. 93, 325-332 (1991).

8. Polifka, J.E., Rutledge, J.C., Kimmel, G.L., Dellarco, V. \& Generoso, W. M. Exposure to ethylene oxide during the early zygotic period induces skeletal anomalies in mouse fetuses. Teratology 53, 1-9 (1996).

\section{Anti-tumor activity of C-raf antisense-correction}

To the editor-In March of 1998, our partner, Novartis Pharmaceuticals, informed us of several cases of data manipulation by a single individual scientist in the Novartis Research Department in Basel, Switzerland. This individual confessed to data manipulation that affected tumor xenograft results obtained with some of our antisense inhibitors, including part of the work presented in our report (Nature Med. 2, 668-675; 1996) describing anti-tumor activity of a phosphorothioate antisense oligodeoxynucleotide targeted against C-raf kinase. The results presented in Figs. 5 and 7 of this article were generated under the supervision of this individual and, as such, may have affected the validity of some of the results described therein. Since the discovery of this incident, Novartis and Isis have jointly and aggressively worked to determine which experiments were affected and to repeat the key experiments as necessary. We can now report the results of that investigation.

Our report described a phosphorothioate oligodeoxynucleotide that in a highly specific manner inhibited expression of C-raf kinase in cell culture and in tumor xenograft models. We reported antiproliferative activity in cell culture and anti-tumor activity in animals against three tumor xenograft models (A549, T24 and MDA.MB231) at doses below $6 \mathrm{mg} / \mathrm{kg}$ per day. We can now confirm that all the in vitro, as well as the in vivo mRNA expression data, is accurate but that the data showing inhibition of tumor growth following administration of the Craf antisense in tumor xenograft models is not (Figs. 5 and 7). That is, we are unable to confirm any in vivo anti-tumor activity of the C-raf antisense molecule under the conditions reported.

We have observed substantial anti-tumor activity against a range of tumor types in xenograft models after administration of the C-raf antisense. However, the doses required to achieve anti-tumor activity in these models are generally higher than that described in the earlier report (10-20 mg/kg compared with $0.6 \mathrm{mg} / \mathrm{kg}$ ).

We deeply regret any confusion that this may have caused the scientific community and are eager to address any issues or problems stemming from our earlier report.

BRETT P. MONIA

Director, Molecular Pharmacology

Isis Pharmaceuticals, 2292 Faraday Ave.

Carlsbad, CA 92008,Email bmonia@isisph.com 